\title{
Influencia del peso vivo y la alimentación sobre el desempeño de terneros en su primer invierno
}

\author{
Carriquiry, E.J.'; Fernández, J.A.'; Yáñez, E.A. ${ }^{3}$ \\ ${ }^{1}$ Actividad privada, Florida, Uruguay. ${ }^{2}$ Dpto. Producción Animal, Fac.Cs.Agrarias, Univ.Nacional Nordeste \\ (UNNE). ${ }^{3}$ Dpto. Prod.Anim., Fac.Cs.Veterinarias, UNNE, \\ Sargento Cabral 2139, Corrientes, Argentina. E-mail: enriquey.mv@gmail.com
}

\begin{abstract}
Resumen
Carriquiry, E.J.; Fernández, J.A.; Yáñez, E.A.: Influencia del peso vivo y la alimentación sobre el desempeño de terneros en su primer invierno. Rev. vet. 27: 1, 14-20, 2016. Con el objetivo de evaluar el aumento de peso vivo (APV) generado por la estrategia de alimentación invernal y la influencia del peso vivo (PV) inicial, se utilizaron 600 terneros de un establecimiento ganadero de Florida, Uruguay. Los animales se distribuyeron en un diseño completo al azar con arreglo factorial, considerando su tamaño (chicos, medianos y grandes, cuyos promedios iniciales de PV fueron 86,114 y $160 \mathrm{~kg}$ ), los cuales se asignaron a 5 dietas con dos niveles energéticos (2,23 y 2,54 Mcal $/ \mathrm{kg}$ de materia seca, MS) y dos fuentes de fibra (henolaje de sorgo y de moha) en animales confinados, así como un lote en pastoreo con suplementación de alta energía. El APV fue de 300 a 600 g/día y de 600 a 800 g/día para dietas de baja y alta energía, respectivamente. La condición corporal al salir del invierno fue de 5,2 y de 4,9 y la eficiencia de conversión fue de 7,7 y 11,1, para los tratamientos de alta y baja energía, respectivamente, existiendo interacción entre peso inicial y tratamientos. El confinamiento de los animales en corrales determinó un desempeño más ajustado al previsto, comparados con aquéllos suplementados a campo. La proporción de fibra utilizada (FDN 36,7 y 47,5\%) fue importante en determinar el APV en confinamiento. Con dietas de alta concentración energética la eficiencia de conversión fue superior en animales más livianos que en aquéllos más pesados, lo cual confirma la mayor sensibilidad de los animales más livianos al tipo de dieta utilizada. Las fuentes de fibra no generaron diferencias en ninguna de las variables evaluadas. La suplementación en pastoreo y el confinamiento demostraron ser alternativas para mejorar el desempeño tradicional de los terneros en su primer invierno.
\end{abstract}

Palabras clave: ternero, nutrición, confinamiento, pastoreo, ganancia de peso.

\begin{abstract}
Carriquiry, E.J.; Fernández, J.A.; Yáñez, E.A.: Influence of live weight and feeding on the development of calves in their first winter. Rev. vet. 27: 1, 14-20, 2016. In order to assess the change in body weight gain (BWG) due to the effects of winter feeding strategy and the influence of initial BW, 600 young calves were included in a study in a farm in Florida, Uruguay. Animals were grouped in a complete random design with factorial arrangement, according to the mean initial BW (86, 114 and $160 \mathrm{~kg})$ and 5 diets: 2 energy levels, low and high (2.23 and $2.54 \mathrm{Mcal} / \mathrm{kg} \mathrm{DM}$ ), and 2 fiber sources, sorghum and moha hay, in confined animals; and grazing plus high-energy supplementation (T5). Daily BWG were from 300 to 600 $\mathrm{g} /$ day and 600 to $800 \mathrm{~g}$ /day for low and high energy diets, respectively. Body condition at the end of the winter was 5.2 and 4.9; the conversion efficiency was 7.7 and 11.1 for treatments of high and low energy, respectively, and there was interaction between initial weight and nutritional level. The confinement of animals determined a tighter performance compared to those supplemented with natural grass with strong forage limitations. The proportion of fiber used (NDF 36.7 y 47.5\%) was important to determine the BWG in confinement. With high energy diets, conversion efficiency was higher in lighter animals compared to heavier animals; this confirms the greater sensitivity of lighter animals to the type of diet used in the assay. Fiber sources did not cause differences for the assessed variables. Supplementa-
\end{abstract}


tion during grazing and confinement proved to be alternatives for improving the traditional performance of young calves in their first winter, allowing animals to obtain the necessary BWG for the next stages of the production system.

Key words: calf, nutrition, feedlot, grazing, liveweight gain.

\section{INTRODUCCIÓN}

Las pasturas naturales de Uruguay, sur de Brasil y nordeste de Argentina son principalmente gramíneas subtropicales de crecimiento primavero-estival, por lo cual presentan una marcada estacionalidad. Según los registros de 18 años de trabajo, la tasa de crecimiento diario promedio mensual de los campos naturales de basamento cristalino de la zona de Cerro Colorado (Florida, Uruguay), no supera los $10 \mathrm{~kg}$ de materia seca (MS/ha/día) del $1^{\circ}$ de abril al 15 de septiembre, siendo julio el mes de menor crecimiento promedio $(5 \mathrm{~kg} \mathrm{MS} /$ ha/día) ${ }^{10}$.

En la zona de Mercedes (Corrientes, Argentina), en diferentes tipos de pastizal natural (pajonal, pastos cortos y flechillar) se reportaron tasas de crecimiento diario inferiores a $10 \mathrm{~kg} \mathrm{MS} / \mathrm{ha}$ en otoño e invierno ${ }^{1,18}$.

Esta deficiencia otoño-invernal en la oferta forrajera afecta el crecimiento de los terneros destetados, causando pérdidas de hasta $40 \mathrm{~kg}$ de peso vivo (PV), desde el destete en otoño hasta fines del invierno ${ }^{20} \mathrm{y}$ hasta el $20 \%$ del PV, con mortandad de animales ${ }^{8}$. En campo natural, con diferentes cargas y disponibilidades de forraje, las pérdidas de peso de los terneros osciló entre 30 y $225 \mathrm{~g} /$ día de $\mathrm{PV}{ }^{14}$.

Varias estrategias de alimentación han sido utilizadas para solucionar esta situación: pastizales mejorados, forrajeras cultivadas, suplementación invernal y, en forma incipiente en los últimos años, el confinamiento estratégico o temporario para la recría de los terneros durante su primer invierno post-destete. En las zonas con mayor deficiencia estacional en la producción de MS, la suplementación proteica es una técnica ampliamente utilizada para mejorar el aprovechamiento del forraje diferido del otoño.

El uso de mejoramientos extensivos de campo natural con especies de clima templado ha incrementado dicha performance en forma moderada, logrando desde mantenimiento hasta buenas ganancias individuales (hasta $800 \mathrm{~g} / \mathrm{día}$ ), pero con una enorme variabilidad entre animales, entre pasturas y entre años.

En terneros en su primer invierno, utilizando pastizales mejorados con Lotus pedunculatus y L. subbiflo$r u s$, con diferentes asignaciones de forraje (desde 3 a 22 $\mathrm{kg}$ MS cada $100 \mathrm{~kg}$ de PV/día), se lograron entre 200 y $700 \mathrm{~g}$ de aumento de PV (APV), detectándose también crecimiento compensatorio en primavera, manifestado por APV significativamente mayor en los animales con menores ganancias invernales ${ }^{2}$.

En terneras pastoreando pastizal natural la suplementación invernal con afrechillo de arroz, sorgo o ex- peller de girasol, ofrecido en cantidades que no superan el 1,5\% del PV, produjo ganancias invernales de 100 a $200 \mathrm{~g} /$ día, situación que permite una buena tasa de ganancia diaria (500 a $700 \mathrm{~g}$ /día) sobre pasturas naturales en primavera ${ }^{19}$. Trabajos realizados en el nordeste argentino revelaron una problemática invernal similar, por la pérdida de calidad de las pasturas subtropicales, con el agravante de la limitación ecológica a la implantación de pasturas templadas con crecimiento invernal ${ }^{4,21,22}$.

Terneros suplementados en invierno con afrechillo de trigo y semilla de algodón fueron evaluados en Chaco (Argentina), demostrándose que con baja disponibilidad forrajera y suplementación energético-proteica al 0,7\% del PV, se obtenían resultados aceptables en la recría invernal, con ganancias de 250 a 275 g/día ${ }^{3}$. En terneros, la suplementación con concentrados energéticos de $75 \%$ de digestibilidad y $14 \%$ de proteína bruta, produjo ganancias entre el 0,7 y $1 \%$ del PV durante 90 a 120 días, logrando cambiar la tendencia de perder a ganar PV y la eficiencia de conversión es de 3 a $1{ }^{23}$.

Resultados experimentales de encierres determinaron APV de 700 a $1.140 \mathrm{~g}$ dependiendo del nivel y tipo de dieta ${ }^{5,13,17}$, concluyéndose que las ventajas de esta técnica consisten en aprovechar la elevada eficiencia de conversión del animal joven, y predecir con mayor precisión los APV de los animales, en momentos en que las ganancias de peso son variables e impredecibles en condiciones de pastoreo.

Si bien el ternero es la categoría que posee la máxima eficiencia de conversión, por lo cual lograr la mayor ganancia en el corral sería beneficioso, se debe considerar el destino de estos animales, así como el efecto sobre la siguiente etapa cuando se prevé que la misma sea en pastoreo.

Este trabajo, realizado entre los meses de junio a setiembre del año 2008, tuvo como objetivo evaluar la variación de los APV debidos a la estrategia de alimentación invernal y la influencia del PV inicial del ternero.

\section{MATERIAL Y MÉTODOS}

El trabajo se llevó a cabo en la Estancia Santa Clara, empresa situada en el Departamento de Florida, República Oriental del Uruguay ( $33^{\circ} 40 \mathrm{~S}$ y $53^{\circ} 55 \mathrm{~W}$ ), sobre suelos de basamento cristalino en una zona netamente ganadera con incipiente desarrollo agrícola.

Se dispuso de 600 terneros machos castrados y trazados (doble caravana, visual y electrónica), nacidos en el mismo establecimiento en la primavera de 2007, destetados precozmente entre los 60 y 90 días de edad, 
con muy bajo PV debido a la intensa sequía del verano 2007-2008. Luego del destete los animales se recriaron a campo hasta el 27/05/2008, cuando comenzó la etapa de acostumbramiento para el presente trabajo.

$\mathrm{Al}$ inicio del ensayo, los animales fueron divididos en tres grupos por su PV promedio: chicos de $86,5 \mathrm{~kg}$, medianos de $114 \mathrm{~kg}$ y grandes de $160,1 \mathrm{~kg}$. Estos animales se distribuyeron en lotes homogéneos de 40 cabezas en cada unidad experimental, y fueron asignados aleatoriamente a cada uno de las cinco dietas, alojándolos en 12 corrales del feedlot o 3 potreros de pastoreo.

En los terneros confinados se ensayaron diferentes dietas, a saber: dieta de baja energía y rollos de sorgo (T1 o baja sorgo), dieta de baja energía y rollos de moha (T2 o baja moha), dieta de alta energía y rollos de sorgo (T3 o alta sorgo), dieta de alta energía y rollos de moha (T4 o alta moha) y terneros en pastoreo con suplementación de alta energía (T5 o alta campo).

Se utilizó un diseño completamente aleatorizado con arreglo factorial de $3 \times 5$, donde se combinaron los tres tamaños de animales según su distinto $\mathrm{PV}$ al inicio del ensayo (chicos, medianos y grandes) y las cinco dietas para su alimentación, considerando a los 40 animales de cada combinación como unidad experimental, reconociendo como pseudo-repeticiones.

Los animales de cada tratamiento en confinamiento se distribuyeron en corrales con superficie de 4.000 $\mathrm{m}^{2}$ con comederos de fibra de vidrio $(50 \mathrm{~cm}$ lineales para cada ternero) y bebederos de hormigón en el centro del corral. El alimento concentrado fue mezclado y distribuido con un mixer por la mañana y la fibra fue distribuida manualmente. Para el T5 se dispuso de tres potreros de similar tamaño (58, 62 y $64 \mathrm{ha})$, con pastizales naturales. El concentrado se ofreció en las mismas condiciones que para los confinados.

Para los tratamientos de encierre a corral las dietas se elaboraron con grano de sorgo ensilado como grano húmedo, expeller de girasol y -como fuentes de fibrahenolaje de planta entera de moha ("rollos de moha") o henolaje de rastrojo de sorgo en silo pack ("rollos de sorgo"). Además se agregó una mezcla granulada como fuente de minerales, vitaminas y monensina (Complemix Iniciador ${ }^{\circledR}$ ).

Las dietas fueron formuladas con base en las tablas de requerimientos del NRC ${ }^{15}$. Los alimentos utilizados fueron analizados en el Laboratorio Analítico Agro Industrial (LAAI) y en el Laboratorio de Servicios Analíticos de Alimental S.A. (Río Cuarto, Argentina). Para ambas dietas, los porcentajes de cada ingrediente son presentados en Tabla 1 y los valores nutricionales en Tabla 2.

Las dietas fueron ajustadas de acuerdo a la evolución del PV de los animales y de acuerdo al resultado obtenido y la expectativa prevista de desempeño: moderado (500 g/día) y alto (1 kg/día); es así que a los animales confinados se les ofreció un $3 \%$ del PV (desempeño moderado) y $3 \%$ del $\mathrm{PV}$ al inicio y $3,6 \%$ a partir del segundo mes de ensayo, para desempeño alto. La oferta fue corregida semanalmente.
Tabla 1. Composición de las dietas utilizadas (base fresca).

\begin{tabular}{lccc}
\hline alimento (\%) & alta energía & baja energía & campo \\
\hline sorgo, grano húmedo & 40,5 & 12,5 & 40,5 \\
girasol, pellets & 18,5 & 19,5 & 18,5 \\
núcleo vitam-mineral & 2 & 2 & 2 \\
rollo (moha o sorgo) & 39 & 66 & 0 \\
pastura natural & 0 & 0 & 39 \\
\hline
\end{tabular}

Tabla 2. Valores nutricionales de las dietas (base seca).

\begin{tabular}{lcc}
\hline variable & baja energía & alta energía \\
\hline proteína bruta (\%) & 14,0 & 13,9 \\
RDP (\% MS) & 11,5 & 10,7 \\
RUP (\% MS) & 2,50 & 3,20 \\
EM (Mcal/kg) & 2,23 & 2,54 \\
ENg (Mcal) & 0,51 & 0,87 \\
FDN (\%) & 47,5 & 36,7 \\
FDNe (\% MS) & 26,2 & 18,5 \\
cenizas (\%) & 10,30 & 7,05 \\
calcio (\%) & 1,29 & 0,98 \\
fósforo total (\%) & 0,53 & 0,53 \\
magnesio (\%) & 0,87 & 0,73 \\
sodio (\%) & 0,27 & 0,22 \\
cobre (ppm) & 24,0 & 20,3 \\
cinc (ppm) & 58,8 & 49,7 \\
ionóforo (ppm) & 38,3 & 32,3 \\
\hline
\end{tabular}

RDP: proteína degradable en rumen, RUP: proteína no degradable en rumen, FDNe: fibra detergente neutro efectiva, EM: energía metabolizable, ENg: energía neta de ganancia.

La cantidad suministrada de concentrado y fuentes de fibra fue medida diariamente para cada tratamiento. En T5 el concentrado se distribuyó diariamente, de acuerdo a las cantidades previstas. La evaluación de la disponibilidad de pastura se realizó el 31 de julio de 2008, por el método Botanal ${ }^{10}$.

Los animales tuvieron un período de acostumbramiento al confinamiento, a la dieta y a la rutina de alimentación que abarcó el lapso del 27 de mayo al 19 de junio de 2008. Durante este período todos los terneros confinados recibieron la misma dieta. El ensayo tuvo una duración de 98 días (19 de junio al 25 de setiembre de 2008), abarcando la totalidad del invierno.

Los animales, identificados con caravanas electrónicas, fueron pesados al inicio del acostumbramiento y luego una vez por mes. La determinación del PV se realizó previo ayuno de por lo menos 6 horas, con balanza electrónica "True-test" regulada al inicio del experimento por un técnico de la empresa importadora.

La evaluación de la condición corporal (CC) fue realizada mediante apreciación visual por un único evaluador, al ingresar a la balanza, previo a la última pesada. La escala utilizada fue de 1 a $8^{11,24}$, siendo 1 el animal más flaco y 8 el más engrasado. La conversión alimenticia o eficiencia de conversión fue calculada considerando los kg de MS suministrada divididos por el APV. 
Los datos se analizaron mediante análisis de varianza (ANOVA) para un diseño completamente aleatorizado con arreglo factorial y posterior prueba de comparación de medias por test de Tukey $(\alpha=0,05)$, para todas las variables evaluadas. En el caso de que la interacción tamaño (animal) por tratamiento (dieta) resultara significativa $(\mathrm{p}<0,05)$ se realizó ANOVA de la misma variable para cada tamaño animal.

En las variables PV y CC a la salida del corral, se agregó al modelo la covariable PV inicial. Todos los análisis estadísticos se realizaron con el software Infostat ${ }^{12}$.

\section{RESULTADOS Y DISCUSIÓN}

El período de acostumbramiento fue analizado de forma independiente en cada tamaño animal, usando el peso inicial como covariable (Tabla 3), debido a que se registraron notorias diferencias de tamaño y estado entre los terneros, por lo que se preveía que la performance ya podía mostrar ya diferencias en esta etapa. Durante este período todos los terneros confinados recibieron la misma dieta, excepto en los 3 lotes que fueron suplementados en el campo (T5), los cuales nunca fueron confinados.

En los terneros chicos hubo diferencias significativas en la performance del acostumbramiento, obteniendo el grupo T5 la mayor ganancia. Los terneros medianos mostraron la misma tendencia, en tanto que en los terneros grandes no hubo diferencias significativas en el rendimiento durante el acostumbramiento, con un coeficiente de variación (CV) muy alto.

El período de evaluación invernal comprendió 98 días (entre el 19 de junio y el 25 de septiembre de 2008). En la Figura 1 se exhiben las ganancias de peso de los terneros de cada tratamiento durante todo el período invernal. Los resultados del ANOVA durante dicho lapso mostraron efecto significativo para el modelo, con un $\mathrm{R}^{2}$ del $60 \%$, existiendo interacción entre tamaño animal y tratamientos, por lo cual se optó por abrir la interacción por los diferentes tamaños. En los tres tamaños de animales considerados, los tratamientos con alta energía lograron mayor APV que T1 y T3, los cuales incluían mayor proporción de fibra (Figura 1).

En el caso de los tratamientos con alto uso de concentrado en los terneros medianos, los animales del grupo T5 tuvieron significativamente mayor APV $(780 \mathrm{~g})$ que aquellos en corral con rollo de sorgo como fibra $(670 \mathrm{~g})$. Esta diferencia, que no se manifestó en los terneros chicos ni grandes, estaría explicada por una mayor disponibilidad de forraje (460 kg de miento.
MS/ha) en comparación a los otros potreros (395 kg de MS/ha). Adicionalmente, la composición botánica del potrero de animales medianos, presentó mayor proporción de gramíneas.

Otra forma de evaluar la performance durante el confinamiento es con el aumento total de PV. Los resultados de los tratamientos y las diferencias estadísticas se mantienen. La ventaja es que, como se aprecia en la Tabla 4, los PV promedio de entrada y salida marcan tendencia del tipo de animal que ingresó y el que sale.

Condición corporal. Los valores obtenidos para esta importante variable (CC) se consignan en Tabla 5. En general no es recomendable un exceso de gordura durante la recría del animal, por su impacto negativo sobre el costo energético y económico, así como la posibilidad del ulterior crecimiento compensatorio de primavera, cuando el animal ha tenido ganancias moderadas durante el período invernal.

El modelo utilizado para evaluar este parámetro consideró el PV inicial como covariable, el cual resultó altamente significativo en todos los tamaños de animal, con bajos coeficientes de variación, entre 5 y $7 \%$, con $\mathrm{R}^{2}=0,23$ a 0,41 . En los terneros chicos, los tratamientos T1 y T2 (baja energía), así como T5, revelaron una CC menor a T3, en tanto T4 superó a T2 -con la misma fuente de fibra- y a T5.

Tabla 3. Evolución del peso $(\mathrm{kg})$ durante el período de acostumbra-

\begin{tabular}{lcccccc}
\hline \multirow{2}{*}{ tamaño } & \multicolumn{2}{c}{ terneros chicos } & \multicolumn{2}{c}{ terneros medianos } & \multicolumn{2}{c}{ terneros grandes } \\
\cline { 2 - 7 } & $\mathrm{PV}$ & $\mathrm{APV}$ & $\mathrm{PV}$ & $\mathrm{APV}$ & $\mathrm{PV}$ & $\mathrm{APV}$ \\
\hline baja sorgo & $85,9 \mathrm{a}$ & $5,90 \mathrm{~b}$ & $118,9 \mathrm{ab}$ & $6,97 \mathrm{ab}$ & $166,2 \mathrm{a}$ & $2,37 \mathrm{a}$ \\
baja moha & $85,1 \mathrm{a}$ & $5,80 \mathrm{~b}$ & $113,2 \mathrm{~b}$ & $5,50 \mathrm{ab}$ & $163,4 \mathrm{a}$ & $2,92 \mathrm{a}$ \\
alta sorgo & $85,1 \mathrm{a}$ & $6,96 \mathrm{ab}$ & $120,6 \mathrm{a}$ & $4,94 \mathrm{~b}$ & $158,4 \mathrm{ab}$ & $2,36 \mathrm{a}$ \\
alta moha & $86,9 \mathrm{a}$ & $5,72 \mathrm{~b}$ & $115,5 \mathrm{ab}$ & $6,37 \mathrm{ab}$ & $166,9 \mathrm{a}$ & $4,80 \mathrm{a}$ \\
alta campo & $89,8 \mathrm{a}$ & $8,80 \mathrm{a}$ & $102,0 \mathrm{c}$ & $8,60 \mathrm{a}$ & $145,8 \mathrm{~b}$ & $4,97 \mathrm{a}$ \\
$\overline{\mathrm{x}}$ grupo & 86,5 & 6,04 & 114,0 & 6,48 & 160,1 & 3,48 \\
CV \% & 14,0 & 68 & 10,0 & 85 & 12,6 & 184 \\
DMS & 7,56 & 2,81 & 7,17 & 3,42 & 12,7 & 4,04 \\
\hline
\end{tabular}

PV: peso vivo inicial, APV: aumento del peso vivo, $\overline{\mathrm{x}}$ : media aritmética, CV: coeficiente de variación, DMS: diferencia mínima significativa. Letras distintas en la misma columna indican diferencias significativas $(p<0,05)$.

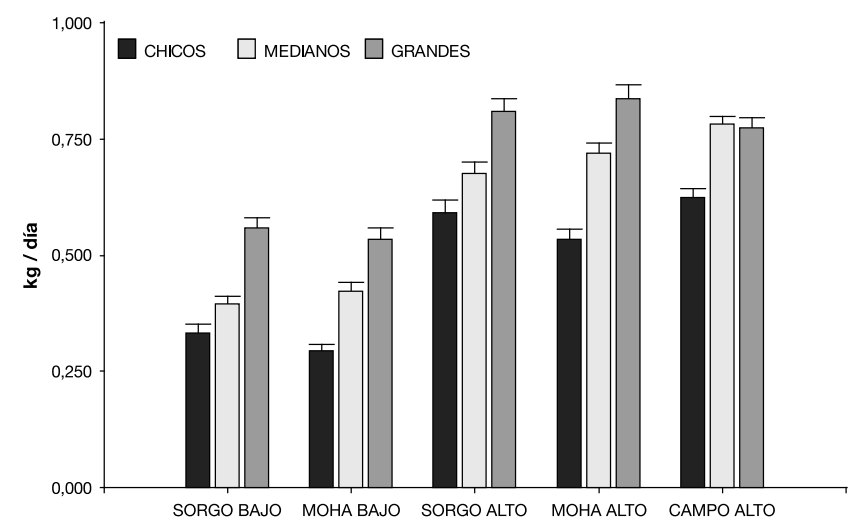

Figura 1. Promedios de aumento de peso de los terneros según tratamiento, durante el período invernal. 
Tabla 4. Promedios de peso vivo $(\mathrm{kg})$ inicial y final (salida de corral) durante el período invernal.

\begin{tabular}{|c|c|c|c|c|}
\hline terneros & tratamiento & PV inicial $^{\mathrm{a}}$ & PV final ${ }^{\mathrm{b}}$ & APV \\
\hline \multirow[t]{8}{*}{ chicos } & baja sorgo & 91,8 & 126,0 & $32,6 \mathrm{C}$ \\
\hline & baja moha & 91,1 & 122,4 & $28,7 \mathrm{C}$ \\
\hline & alta sorgo & 92,2 & 151,4 & $58,1 \mathrm{AB}$ \\
\hline & alta moha & 92,6 & 145,5 & $52,2 \mathrm{~B}$ \\
\hline & alta campo & 98,2 & 153,0 & $61,1 \mathrm{~A}$ \\
\hline & $\bar{x}$ & 93,2 & 139,7 & 46,5 \\
\hline & $\mathrm{CV}$ & $12,4 \%$ & $13,8 \%$ & $27,9 \%$ \\
\hline & DMS & - & - & 8,15 \\
\hline \multirow[t]{8}{*}{ medianos } & baja sorgo & 118,9 & 159,1 & $38,6 \mathrm{C}$ \\
\hline & baja moha & 113,2 & 162,1 & $41,4 \mathrm{C}$ \\
\hline & alta sorgo & 120,6 & 186,7 & $66,2 \mathrm{~B}$ \\
\hline & alta moha & 115,5 & 191,1 & $70,5 \mathrm{AB}$ \\
\hline & alta campo & 102,0 & 197,4 & $76,6 \mathrm{~A}$ \\
\hline & $\bar{x}$ & 120,5 & 179,2 & 58,7 \\
\hline & $\mathrm{CV}$ & $9,6 \%$ & $9,6 \%$ & $21,0 \%$ \\
\hline & DMS & - & - & 7,7 \\
\hline \multirow[t]{8}{*}{ grandes } & baja sorgo & 166,6 & 217,8 & $54,8 \mathrm{~B}$ \\
\hline & baja moha & 163,4 & 215,6 & $52,4 \mathrm{~B}$ \\
\hline & alta sorgo & 158,4 & 243,0 & $79,3 \mathrm{~A}$ \\
\hline & alta moha & 166,9 & 244,9 & $82,1 \mathrm{~A}$ \\
\hline & alta campo & 145,8 & 240,3 & $75,8 \mathrm{~A}$ \\
\hline & $\bar{x}$ & 163,7 & 232,6 & 68,9 \\
\hline & $\mathrm{CV}$ & $12,2 \%$ & $11,4 \%$ & $22,3 \%$ \\
\hline & DMS & - & - & 9,6 \\
\hline
\end{tabular}

PV: peso vivo, APV: aumento del PV, ${ }^{a} 19$ de junio; ${ }^{b} 25$ de setiembre (año 2008), $\bar{x}$ : media aritmética, CV: coeficiente de variación, DMS: diferencia mínima significativa $(\mathrm{kg})$. Letras distintas dentro de cada bloque indican diferencias significativas $(\mathrm{p}<0,05)$.

Tabla 5. Condición corporal (escala 1 a 8) al final del período evaluado.

\begin{tabular}{lcccc}
\hline tratamiento & chicos & medianos & grandes & $\overline{\mathrm{x}}$ tratamiento \\
\hline baja sorgo & $4,80 \mathrm{BC}$ & $4,84 \mathrm{~B}$ & $5,10 \mathrm{BC}$ & 4,91 \\
baja moha & $4,70 \mathrm{C}$ & $4,81 \mathrm{~B}$ & $5,03 \mathrm{C}$ & 4,85 \\
alta sorgo & $5,09 \mathrm{~A}$ & $5,12 \mathrm{~A}$ & $5,41 \mathrm{~A}$ & 5,21 \\
alta moha & $5,01 \mathrm{AB}$ & $5,15 \mathrm{~A}$ & $5,45 \mathrm{~A}$ & 5,20 \\
alta campo & $4,74 \mathrm{C}$ & $5,11 \mathrm{~A}$ & $5,22 \mathrm{~B}$ & 5,02 \\
$\overline{\mathrm{X}}$ grupo & 4,87 & 5,01 & 5,24 & $\overline{\mathrm{x}}$ general $=5,04$ \\
DMS & 0,216 & 0,182 & 0,183 & - \\
\hline
\end{tabular}

Letras distintas en la misma columna indican diferencias significativas $(\mathrm{p}<0,05)$. DMS: mínima diferencia significativa.

En los terneros medianos los grupos de corral de baja energía ostentaron una $\mathrm{CC}$ menor a todos los tratamientos de alta energía. En los terneros grandes nuevamente se repitió la diferencia de los grupos de alta energía en corral frente al resto, quedando el lote de alta energía a campo con un resultado intermedio.

En general estos resultados guardan coherencia con el superior APV de estos animales en todo el período, a excepción del tratamiento T5 para los animales chicos y grandes, que no se diferenciaron de los tratamientos
Tabla 6. Eficiencia de conversión ( $\mathrm{kgMS} / \mathrm{kgAPV}$ ) según tratamiento y tamaño del animal.

\begin{tabular}{lcccc}
\hline tratamiento & chicos & medianos & grandes & $\overline{\mathrm{x}}$ tratam. \\
\hline baja sorgo & $11,99 \mathrm{~A}$ & $11,31 \mathrm{~A}$ & $10,40 \mathrm{~A}$ & $11,24 \mathrm{~A}$ \\
baja moha & $11,80 \mathrm{~A}$ & $10,38 \mathrm{~A}$ & $10,88 \mathrm{~A}$ & $11,02 \mathrm{~A}$ \\
alta sorgo & $6,85 \mathrm{~B}$ & $7,73 \mathrm{~B}$ & $8,20 \mathrm{~B}$ & $7,59 \mathrm{~B}$ \\
alta moha & $7,59 \mathrm{~B}$ & $7,28 \mathrm{~B}$ & $8,40 \mathrm{~B}$ & $7,76 \mathrm{~B}$ \\
$\overline{\mathrm{x}}$ bloque & 9,56 & 9,18 & 9,47 & 9,40 \\
CV \% & 63 & 34 & 25 & 44 \\
DMS & 3,49 & 1,80 & 1,42 & 1,40 \\
\hline
\end{tabular}

Letras distintas en la misma columna indican diferencias significativas $(\mathrm{p}<0,05)$. DMS: mínima diferencia significativa.

de baja energía. Solo se diferenciaron los terneros medianos, circunstancia atribuible a la oferta forrajera, como ya fuera explicado.

Mayores niveles de engrasamiento en terneros recriados a corral fueron observados en los animales con mayores ganancias diarias de peso ${ }^{6}$, circunstancia que se explica por un mayor nivel de consumo. Cuanto mayor fue la condición corporal al inicio, tanto más pesados fueron los terneros al final; el ascenso de la CC se explica porque los más altos APV generaron mayores deposiciones de tejido adiposo.

Eficiencia de conversión. Esta característica se define como la cantidad de alimento por unidad de aumento de $\mathrm{PV}{ }^{9}$. Cabe aclarar que el grupo T5 no fue considerado para esta variable, porque no hubo medición de consumo de MS del forraje que pastoreaban los animales. En este trabajo no se midió el consumo de los animales confinados, sino que se utilizó como estimador del mismo la cantidad de alimento ofrecido al lote dividido los animales de cada corral.

En la Tabla 6 se puede observar que el efecto del tratamiento fue significativo, logrando una mayor eficiencia de conversión en las dietas de alta energía frente a los tratamientos con baja energía.

Este resultado puede atribuirse a que, ante requerimientos similares de los terneros para mantenimiento, un alimento con mayor concentración energética aporta más nutrientes disponibles para ganancia de PV, por lo que ante un consumo levemente mayor $(20 \%)$, los APV (Tabla 4) fueron superiores (entre 45 y $85 \%$ ).

Sin embargo, se destaca que no hubo efecto significativo del tamaño del animal ( $\mathrm{p}=0,70)$, lo cual contrasta con varios trabajos que citan que cuanto más pequeño es el animal más eficiente es. Hubo diferencias importantes en el CV entre los tamaños de los animales, quizás sugiriendo que los más chicos sufren más la baja calidad de la dieta.

En la Figura 2 se observa que cuanto más liviano el animal, la pérdida de eficiencia es mayor al usar dietas con alta proporción de fibra, menor digestibilidad y menor concentración energética.

Con dietas de alta concentración energética, más grano y menos fibra, los animales más livianos tienden 
a ser más eficientes que los pesados. Los terneros con menor PV inicial fueron más sensibles a la calidad del alimento durante el confinamiento, lo cual indica que esta estrategia para la recría exige asegurar determinada calidad de alimento para obtener resultados adecuados y predecibles. Por otra parte se deduce que cuando se dispone de dietas de alta concentración energética, cuanto más pesados sean los terneros menor será la eficiencia y mayor la cantidad de alimento por $\mathrm{kg}$ de PV producido.

A su vez, en la Figura 2 se puede observar que los animales con mayor PV inicial, se adaptaron mejor a las dietas de menor calidad, con mayor eficiencia de conversión. La necesidad de conocer el APV obtenido con las diferentes dietas propuestas, se asocia a la posibilidad de aplicar una restricción moderada al crecimiento durante el primer invierno de recría, con la expectativa de aprovechar el crecimiento compensatorio en una etapa posterior.

El crecimiento compensatorio es definido como el rápido incremento en la tasa de crecimiento relativo a la edad, y es exhibido por los animales alimentados en forma adecuada a sus requerimientos, luego de un período de restricción nutricional suficiente para disminuir su desarrollo continuo ${ }^{16}$. Es una estrategia para manejar costos y, en condiciones de campo dependientes de la variabilidad climática, es una alternativa para incrementar la productividad de animales en pastoreo, al aprovechar la ventaja de una mayor eficiencia del uso de la dieta por la capacidad de compensación de su PV.

Es frecuente la búsqueda de la ganancia mínima en una etapa de alimentación de alto costo (corral en invierno), que puede ser compensada en una etapa posterior de abundancia de forraje de bajo costo (primaveraverano), y podría convertirse en un objetivo clave en la ganadería comercial. Tanto el tamaño inicial como la $\mathrm{CC}$, influyen en gran medida en el resultado económico de la empresa ${ }^{7}$.

El análisis económico de cualquier tecnología puede realizarse en modo parcial o aplicado en un sistema, pero en cualquiera de los casos es una condición indispensable para el desarrollo de la misma.

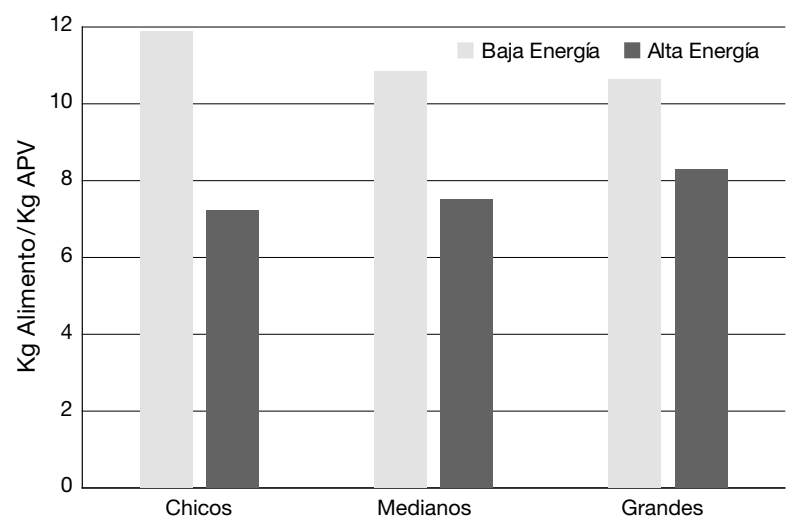

Figura 2. Eficiencia de conversión ( $\mathrm{kg}$ de alimento / $\mathrm{kg}$ de aumento de peso vivo) en función del tamaño del animal y de la dieta utilizada.
Se concluye que el confinamiento de los terneros en su primer invierno permite un desempeño más predecible que en aquéllos suplementados a campo, con mayor APV para las dietas con menor proporción de fibra. Las fuentes de fibra utilizadas no produjeron diferencias en ninguna de las variables evaluadas. Las cinco estrategias de alimentación evitaron las pérdidas de PV que se observan en los terneros en su primer invierno pos-destete pastoreando pastizales naturales.

Agradecimiento. Al Ing. Agr. Pablo Bonasso Gallinal por poner a disposición su Estancia Santa Clara, animales, instalaciones y personal, así como por su colaboración en el trabajo de campo.

\section{REFERENCIAS}

1. Arias LM. 2006. Controles climáticos de la productividad primaria de pastizales de la Provincia de Corrientes. Tesis, Universidad de Buenos Aires, 51 p. Disponible en: http:// www.agro.uba.ar/users/garbulsk/pdfs/Arias_Tesiscompleta_.pdf

2. Ayala W, Bermúdez R, Soca P, et al. 2003. Oferta de forraje de mejoramiento de campo y performance de la recría vacuna. Anales del Seminario "Producción de carne vacuna y ovina de calidad”, INIA, Treinta y Tres, Uruguay, p 69-82.

3. Balbuena O, Kucseva C, Slanac A, Koza G, Schreiner J, Navamuel M, Stahringer R, D'agostini A. 2002. Suplementación invernal de destetes con una mezcla de semilla de algodón y pellets de afrechillo de trigo suministrado seis y tres veces por semana. Disponible en: www.unne. edu.org/cyt/2002/04-Veterinarias/V-028.pdf.

4. Balbuena, O. 2002. Suplementación de destetes durante su primer invierno y de la vaquilla para primer servicio en el NEA. Anales III Seminario de Pasturas y Suplementación Estratégica en Ganado Bovino, Asunción, Paraguay.

5. Barbarosa R, Kugler N, Garcilazo G, Elizalde JC. 2002. Uso de heno entero y picado en la recría de vaquillonas Rev Arg Prod Anim 22: 75-76.

6. Ceconi I, Davies P, Méndez D, Buffarini M, Elizalde J. 2008. Efecto del nivel de engrasamiento inicial y de la ganancia de peso sobre el engrasamiento final de terneros recriados a corral. Memoria Técnica 2007-2008 de la EEA INTA Gral. Villegas, Argentina, p. 69-74.

7. Ceconi I, Davies P, Méndez DG, Elizalde JC, Buffarini MA. 2010. El nivel de engrasamiento inicial y la ganancia de peso durante la recría a corral afectan los resultados físicos y económicos del proceso de invernada. Rev Arg Prod Anim 30: 51-68.

8. Cibils R, Fernández E, Acosta Y. 2002. Suplementación estratégica de la recría vacuna. Disponible en http//:www. inia.org.uy.

9. Di Marco, O. 2009. Eficiencia de utilización del alimento en vacunos. On line: www.inta.gov.ar/Balcarce/info/documentos/ganadería/bovinos/nutrición/efiAliment.

10. Formoso D, Pereira D. 2008. Efecto del pastoreo mixto sobre la vegetación del campo natural en Cristalino Central (Región Centro-Sur, Brasil). Prod. Ovina 20:5-20. 
11. INIA (Instituto Nacional de Investigación y Tecnología Agraria y Alimentaria, Uruguay). 2006. Cartilla de estado corporal vacuno. Versión electrónica disponible en http:// www.inia.org.uy/online/site/publicacion-ver.php?id=1251

12. Infostat. 2007. Versión 1.1, Grupo Infostat, Facultad de Ciencias Agrarias, Universidad Nacional de Córdoba. Argentina.

13. Kugler N, Barbarosa R, Garcilazo G, Elizalde JC. 2002. Utilización de heno picado y entero en la recría de terneras. Rev Arg Prod Anim 22: 76-77.

14. Lagreca M, Medero P, Rattín A. 2008 El confinamiento de terneros como alternativa de alimentación invernal. Tesis de Ingeniería Agronómica, Universidad de la Republica, Montevideo, Uruguay.

15. National Research Council (NRC). 1984. Nutrient requirement of beef cattle, $6^{\circ}$ ed., National Academy Press, Washington, USA, $103 \mathrm{p}$

16. Ojeda A, Molina F, Carmona D. 2007. Crecimiento compensatorio. Una estrategia de manejo de la disponibilidad de pasturas. Anales XI Seminario manejo y utilización de pastos y forrajes en sistemas de producción animal. On line: http://www.avpa.ula.ve/eventos/xi_seminario/Conferencias/Articulo-4.pdf

17. Parra V, Riffel S, Elizalde JC. 2006. Estrategias de inclusión del corral en los sistemas ganaderos de la Argentina, Ed. Gráfica Máxima, Buenos Aires, 179 p.
18. Pizzio RM, Royo Pallares O, Fernández JG, Benitez CA. 2001. Tasa de crecimiento y producción anual de tres pastizales del centro de la Provincia de Corrientes. Anales Primer Congreso Nacional sobre Manejo de Pastizales Naturales, San Cristóbal (Santa Fé, Argentina), p. 49.

19. Quintans G, Vaz Martins D et al. 1993. Efecto de la suplementación invernal sobre el comportamiento de terneras. En: Campo natural: estrategia invernal y suplementación - Resultados experimentales. Publ. INIA, Treinta y Tres, Uruguay, p. 35-51.

20. Quintans G. 2002. Manejo de la recría vacuna en sistemas ganaderos. Anales Seminario de Actualización Técnica: Cría y Recría Ovina y Vacuna, Serie de Actividades de Difusión No 288, INIA, Uruguay, p. 47-56.

21. Rochinotti D. 2002. Cadena de la carne vacuna. Tecnologías para nuevos escenarios, IDIA XXI, INTA Mercedes, Corrientes, 2: 64-68.

22. Sampedro D, Vogel O, Celser R. 2004. Suplementación de vacunos en pastizales naturales. Publ. Serie Técnica $\mathrm{N}^{\mathrm{o}}$ 34. INTA Mercedes, Corrientes (Argentina).

23. Simeone A. 2007. Suplementación energética y proteica en condiciones de pastoreo. Anales Seminario Suplementación y Engorde a Corral, Young (Río Negro, Argentina), p. $57-86$.

24. Vizcarra JA, Ibáñez W, Orcasberro R. 1986. Repetibilidad y reproducibilidad de dos escalas para estimar condición corporal de vacas Hereford. Investigaciones Agronómicas CIAAB-MAP (Uruguay) 7: 45-47.

\section{Revista Veterinaria ingresa a SciELO}

Scientific Electronic Library Online

Revista Veterinaria, publicación oficial de la Facultad de Ciencias Veterinarias de la Universidad Nacional del Nordeste (Corrientes, Argentina), ha logrado acceder al Núcleo Básico de Revistas Científicas Argentinas (Nivel 1), luego de calificar adecuadamente en el Centro Argentino de Información Científica y Tecnológica (CAICYT), según Resolución 2485/14 del Consejo Nacional de Investigaciones Científicas y Técnicas (CONICET).

Sobre un puntaje máximo de 33 se obtuvieron 32 puntos. Tal calificación constituye "una garantía de la excelencia de la publicación" (sic) y queda expedita la vía del Portal SciELO (Scientific Electronic Library Online) para los artículos publicados.

En tal calificación gravitó positivamente la circunstancia de haber aumentado el índice de impacto (Scimago-Elsevier) y haber disminuido las autocitaciones. También se tuvieron en cuenta aspectos como la amplia cobertura de la revista, la calidad científica del Comité Editorial, los criterios de evaluación de los artículos, el origen de los autores (locales $60 \%$, nacionales $13 \%$, extranjeros $26 \%$, en idioma inglés), el adecuado balance entre trabajos científicos originales y reseñas bibliográficas (ambos con alta calidad), así como el estricto cumplimiento de la periodicidad semestral y la favorable acogida por indizadores como Cab, Doaj, Ebsco, Gale Cengage, Infocyt, Latindex y Scopus.

Se consolida de esta manera la continuidad de "Revista Veterinaria", que en su acontecer supera 50 años de existencia en nuestra Facultad, la cual en 2016 cumplió el $96^{\circ}$ aniversario de su fundación. 